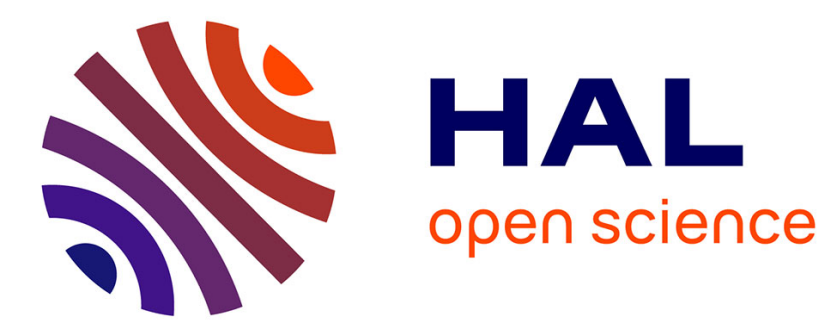

\title{
On some rigidity properties of mappings between CR-submanifolds in complex space
}

\author{
Francine Meylan, Nordine Mir, Dmitri Zaitsev
}

\section{To cite this version:}

Francine Meylan, Nordine Mir, Dmitri Zaitsev. On some rigidity properties of mappings between CRsubmanifolds in complex space. Journées Equations aux Dérivées partielles, Forges-les-Eaux, 2003, Exposé XII. hal-00261700

\section{HAL Id: hal-00261700 https://hal.science/hal-00261700}

Submitted on 8 Mar 2008

HAL is a multi-disciplinary open access archive for the deposit and dissemination of scientific research documents, whether they are published or not. The documents may come from teaching and research institutions in France or abroad, or from public or private research centers.
L'archive ouverte pluridisciplinaire HAL, est destinée au dépôt et à la diffusion de documents scientifiques de niveau recherche, publiés ou non, émanant des établissements d'enseignement et de recherche français ou étrangers, des laboratoires publics ou privés. 


\title{
RIGIDITY PROPERTIES OF MAPPINGS BETWEEN CR-SUBMANIFOLDS IN COMPLEX SPACE
}

\author{
FRANCINE MEYLAN, NORDINE MIR, AND DMITRI ZAITSEV
}

\begin{abstract}
We survey some recent results on holomorphic or formal mappings sending real submanifolds into each other in complex space.
\end{abstract}

\section{INTRODUCTION}

The classical system of Cauchy-Riemann equations in $\mathbb{C}^{n}$ is an important example of an overdetermined system with infinite-dimensional space of solutions. Understanding its "boundary version" (obtained by restricting to real hypersurfaces, or more generally to CR-submanifolds) played an important role in understanding properties of general linear first order PDE systems (see e.g. [Tr00] for a nice exposition on that matter). In contrast to the classical case, in the "boundary case", locally one obtains infinite-dimensional families of nonequivalent PDE systems (corresponding to different CR-structures) that can't be obtained from each other by a change of coordinates. This new phenomenon discovered by Poincaré [Po07] and further studied in seminal work of Cartan [C32a, C32b], Tanaka [Ta62] and Chern-Moser [CM74], attracted recently considerable attention due to its connection to classical theory of PDEs and also to many other extensively developing areas such as e.g. differential, algebraic and symplectic geometry. The relevant system of PDEs for isomorphisms between different tangential Cauchy-Riemann systems is, in the "generic" situation, an overdetermined quasilinear PDE system whose space of solutions (called CR-mappings) may range from zero-dimensional (or even empty) to infinite-dimensional, depending on degeneracies of the systems. The present survey is devoted to recent results on various existence and convergence properties of solutions for these systems. One basic difficulty arising in this context is that the relevant degeneracies may appear for arbitrarily high order derivatives of the system coefficients. Another basic difficulty comes from the presence of singularities that, again, may arise at arbitrarily high order. In $\S 3$ we prove a new result on existence of algebraic solu1tions of algebraic systems (with possible singularities) provided analytic solutions exist. For further discussion of related questions and previously known results we refer the reader to [V85, BN90, Fo93, BER99a, BER00b, Hu01, Z02, R03].

An appendix can be found at the end of the paper, where some notation and basic material needed for this survey are given.

2000 Mathematics Subject Classification. 32H02, 32V20, 32V40

The first author was partially supported by Swiss NSF Grant 2100-063464.00/1. 


\section{EXISTENCE OF CR-MAPPINGS}

We begin by discussing the equivalence problem for real-analytic CR-structures. The usual procedure for treating this problem is to pass to its complexification as follows. It is well-known (see e.g. [BER99a]) that a real-analytic CR-structure can be locally realized via an embedding into a complex space and two such CR-structures are analytically CR-equivalent if and only if the two embeddings are biholomorphically equivalent, i.e. mapped into each other by a biholomoprhic map. Hence we are led to the following basic problem: Given two (germs of) real-analytic submanifolds in $\mathbb{C}^{N}$, when is there a (germ of a) biholomorphic map sending one submanifold into another? In their celebrated paper [CM74], Chern and Moser constructed a normal form for Levi-nondegenerate realanalytic hypersurfaces in $\mathbb{C}^{N}$ providing a solution to the problem for hypersurfaces of this kind. Their construction of the normal form has two parts: a simpler one leading to a formal normal form and a finer geometric construction of the same normal form proving that the obtained normal form is actually convergent when the hypersurface is real-analytic and showing that the formal transformation taking the real hypersurface into its normal form is convergent in this case. In particular, they show in [CM74, §3] that any formal biholomorphic map sending a (germ of a) real-analytic Levi-nondegenerate hypersurface $M \subset \mathbb{C}^{N}$ into another real-analytic Levi-nondegenerate hypersurface $M^{\prime} \subset$ $\mathbb{C}^{N}$ is convergent. We recall that a formal biholomorphic map (or formal equivalence) $f:\left(\mathbb{C}^{N}, p\right) \rightarrow\left(\mathbb{C}^{N}, p^{\prime}\right), p \in M, p^{\prime} \in M^{\prime}$, is an invertible formal holomorphic map (i.e. given by formal power series in $\left.\left(z_{1}, \ldots, z_{N}\right)\right)$ for which the pullback under $f$ of any local real-analytic defining function for $M^{\prime}$ near $p^{\prime}$ vanishes on $M$ (as a formal power series in $\left.\left(z_{1}, \ldots, z_{N}, \bar{z}_{1}, \ldots, \bar{z}_{n}\right)\right)$. Hence the formal equivalence of real-analytic Levi-nondegenerate hypersurfaces in $\mathbb{C}^{N}$ implies their biholomorphic equivalence.

Thus, in case of Levi-nondegenerate hypersurfaces, one can conclude their equivalence by fulfilling the compatibility conditions for CR-mappings at the formal level. More generally, it is natural to ask whether such a property holds for any pair of real-analytic submanifolds $M, M^{\prime} \subset \mathbb{C}^{N}$ of the same dimension. Surprisingly, Moser and Webster [MW83] have produced an example of a (germ of a) two-dimensional real-algebraic surface $M_{0}$ in $\mathbb{C}^{2}$ that is formally equivalent to a real-analytic surface $M_{0}^{\prime} \subset \mathbb{C} \times \mathbb{R}$, but that cannot be biholomorphically mapped (as a germ) to it. Such a surface $M_{0} \subset \mathbb{C}_{z, w}^{2}$ is explicitly given by $w=|z|^{2}+\gamma \bar{z}^{2}+\gamma z^{3} \bar{z}$ for a suitably chosen real number $\gamma>2$. More recently, Gong [G02] gave another class of such examples by showing that, for some $\gamma>2$, the quadric $Q_{\gamma} \subset \mathbb{C}_{z, w}^{2}$ given by $w=|z|^{2}+\gamma\left(z^{2}+\bar{z}^{2}\right)$ is formally but not biholomorphically equivalent to some real-analytic surface in $\mathbb{C}^{2}$.

The example of Moser-Webster leads to the question of deciding for which germs of realanalytic submanifolds the notions of formal and biholomorphic equivalence coincide. More precisely, one has the following problem: Given two real-analytic submanifolds $M, M^{\prime} \subset$ $\mathbb{C}^{N}$ of the same dimension, characterize those points $p \in M$ and $p^{\prime} \in M^{\prime}$ for which formal equivalence of the germs $(M, p)$ and $\left(M^{\prime}, p^{\prime}\right)$ implies their biholomorphic equivalence. A

recent result along these lines in [BRZ01a] states that notions of formal and biholomorphic 
equivalence coincide at all points of nonempty Zariski open subsets of the submanifolds (by Zariski open subsets we shall always mean complements of real-analytic subvarieties). More precisely, the following holds:

Theorem 1.1 (Baouendi, Rothschild, Zaitsev [BRZ01a]). Let $M \subset \mathbb{C}^{N}$ be a connected real-analytic submanifold. Then there exists a closed proper real-analytic subvariety $V \subset$ $M$ such that for any $p \in M \backslash V$, any second real-analytic submanifold $M^{\prime} \subset \mathbb{C}^{N}$ with $\operatorname{dim}_{\mathbb{R}} M=\operatorname{dim}_{\mathbb{R}} M^{\prime}$, and any $p^{\prime} \in M^{\prime}$, the germs $(M, p)$ and $\left(M^{\prime}, p^{\prime}\right)$ are formally equivalent if and only if they are biholomorphically equivalent.

The subvariety $V$ in Theorem 1.1 is explicitly described as a union of three distinguished proper real-analytic subvarieties. The first subvariety is the set of points of $M$ that are not CR (see the appendix for the definition). After removing this subvariety from $M$, we may assume that $M$ is itself $\mathrm{CR}$. To describe the second subvariety, we need to recall the notion of local CR-orbits in a real-analytic CR-submanifold. Given a point $p \in M$, its CR-orbit is the (unique) germ of a real-analytic submanifold $\Sigma^{p}$ through $p$ that satisfies $T_{q} \Sigma^{p} \otimes \mathbb{C}=\mathfrak{g}_{M}(q)$ for all $q \in \Sigma^{p}$, where $\mathfrak{g}_{M}(q)$ denotes the Lie algebra (evaluated at $q$ ) generated by $(1,0)$ and $(0,1)$ vector fields tangent to $M$. The existence and uniqueness of $\Sigma^{p}$ follows from a theorem of Nagano [N62]. The second subvariety to be removed from $M$ consists of those points $p \in M$ for which the map $U \ni q \mapsto \operatorname{dim}_{\mathbb{R}} \Sigma^{q}$ is not constant in any neighborhood $U$ of $p$. Finally, the third subvariety to be removed from $M$ consists of those points that are not of constant degeneracy. A point $p \in M$ is said to be of constant degeneracy if there exists $r \in\{0, \ldots, N-1\}$ such that $M$ is biholomorphically equivalent in a neighborhood of $p$ to a product of the type $\widetilde{M} \times \mathbb{C}^{r}$ for some real-analytic CR-submanifold $\widetilde{M} \subset \mathbb{C}^{N-r}$ satisfying a suitable nondegeneracy condition called finite nondegeneracy (see e.g. [BER99a] for the definition). It can be shown that the set of points of constant degeneracy on a real-analytic CR-submanifold is indeed an (analytic) Zariski open subset of $M$ (see [BRZ01a]).

One natural question that arises from the above discussion is to find the optimal subvariety $V$ for the conclusion of Theorem 1.1 to hold. Since the only known examples of point $p \in M$ for which the conclusion of Theorem 1.1 fails to hold are non CR-points, one may be tempted to conjecture that the subvariety $V$ in Theorem 1.1 is contained in the set of non CR-points:

Conjecture 1.2. Let $M, M^{\prime} \subset \mathbb{C}^{N}$ be real-analytic CR-submanifolds of the same dimension. Then for every $p \in M$, every $p^{\prime} \in M^{\prime}$, the germs $(M, p)$ and $\left(M^{\prime}, p^{\prime}\right)$ are formally equivalent if and only if they are biholomorphically equivalent.

A more recent result supporting this conjecture is the following:

Theorem 1.3 (Baouendi, Mir, Rothschild [BMR02]). Let $M \subset \mathbb{C}^{N}$ be a real-analytic $C R$ submanifold. Then for any point $p \in M$ of finite type, any real-analytic CR-submanifold $M^{\prime} \subset \mathbb{C}^{N}$ with $\operatorname{dim}_{\mathbb{R}} M=\operatorname{dim}_{\mathbb{R}} M^{\prime}$, and any $p^{\prime} \in M^{\prime}$, the germs $(M, p)$ and $\left(M^{\prime}, p^{\prime}\right)$ are formally equivalent if and only they are biholomorphically equivalent. 
Here we recall that a real-analytic CR-submanifold $M \subset \mathbb{C}^{N}$ is of finite type at a point $p \in M$ (in the sense of Kohn [K72] and Bloom-Graham [BG77]) if the Lie algebra generated by all $(1,0)$ and $(0,1)$ vector fields tangent to $M$ spans the complexified tangent space of $M$ at $p$. It is worth mentioning that Theorems 1.1 and 1.3 are independent and not contained into each other. Indeed, given a real-analytic CR-submanifold $M$, the set of points of finite type can be empty, e.g. for $M=M_{0} \times \mathbb{R} \subset \mathbb{C}^{N}$ with $M_{0} \subset \mathbb{C}^{N-1}$ being any CR-submanifold. On the other hand, for the class of real-analytic CR-submanifolds that are of finite type (for which the conclusion of Theorem 1.3 holds at every point), the subvariety $V$ constructed in Theorem 1.1 can be still non-empty:

Example 1.4. Consider the (real-algebraic) hypersurface $M \subset \mathbb{C}_{z_{1}, z_{2}, z_{3}}^{3}$ given by

$$
M:=\left\{\left(z_{1}, z_{2}, z_{3}\right) \in \mathbb{C}^{3}: \operatorname{Im} z_{3}=\left|z_{1} z_{2}\right|^{2}\right\} .
$$

Then $M$ is of finite type through every point, but the subvariety $V$ constructed in Theorem 1.1 is not empty since it can be shown that it is given by the real line $V=$ $\left\{\left(z_{1}, z_{2}, z_{3}\right) \in \mathbb{C}^{3}: \operatorname{Im} z_{3}=z_{1}=z_{2}=0\right\}$. Similarly, for any positive integer $n$, any real-algebraic hypersurface $\widetilde{M} \subset \mathbb{C}^{n+3}$ of the form $\widetilde{M}:=M \times \mathbb{C}^{n}$ is also of finite type and has a non-empty corresponding real-analytic subvariety $V$.

In the next example, we give a situation where Theorems 1.1 and 1.3 cannot be applied to some points.

Example 1.5. Let $M \subset \mathbb{C}^{N}$ be any infinite type real-analytic hypersurface at the origin. Then there exists local holomorphic coordinates $\left(z^{\prime}, z_{N}\right) \in \mathbb{C}^{N-1} \times \mathbb{C}$ near the origin such that $M$ is given by

$$
M:=\left\{\left(z^{\prime}, z_{N}\right) \in \mathbb{C}^{N}: \operatorname{Im} z_{N}=\left(\operatorname{Re} z_{N}\right) \theta\left(z^{\prime}, \overline{z^{\prime}}, \operatorname{Re} z_{N}\right)\right\}
$$

for some real-analytic function $\theta$ (see e.g. [BER99a]). If $M$ is not Levi-flat, then for any point $z \in M \backslash S$, where $S$ is the complex hyperplane of $\mathbb{C}^{N}$ given by $\left\{z_{N}=0\right\}$, $M$ is of finite type at $z$, and Theorem 1.3 can therefore be applied at those points. However, it is an open problem to decide whether the conclusions of Theorems 1.1 and 1.3 also hold for points on the hyperplane $S$.

It is worth pointing out that, in the situation either of Theorem 1.1 or of Theorem 1.3, the assumptions on the manifolds do not imply that all formal equivalences between them converge. Indeed, for instance if $M$ is a product of the form $\widetilde{M} \times \mathbb{C}$ for an arbitrary real-analytic CR-submanifold $\widetilde{M}$, then for any point $p \in M$, it is easy to construct nonconvergent formal self-maps of $M$ centered at $p$. We shall see in $\S 4$ that more restrictive assumptions have to be put on the manifolds to obtain convergence of all their formal equivalences.

Although the proofs of Theorems 1.1 and 1.3 are very different, they both use the approximation theorem due to Artin [A68] (or its generalization). We recall it for the convenience of the reader. 
Theorem 1.6. (Artin [A68]) Let $S(x, y)=\left(S_{i}(x, y)\right)_{i \in I}, S_{i}(x, y) \in \mathbb{K}\{x, y\}$, be a family of convergent power series, where $\mathbb{K}=\mathbb{R}$ or $\mathbb{C}, x \in \mathbb{K}^{n}, y \in \mathbb{K}^{p}$. Suppose that there exist a formal solution $y(x) \in(\mathbb{K}[[x]])^{p}, y(0)=0$, of the system of equations

$$
S(x, y)=0 .
$$

Then for any nonnegative integer $k$, there exists a convergent solution of the system (1.1), for which the Taylor series agrees with that of $y(x)$ up to order $k$.

We should mention that Theorem 1.1 and Theorem 1.3 are correspondingly consequences of the following more general approximation results:

Theorem 1.7. (Baouendi, Rothschild, Zaitsev [BRZ01a]) Let $M \subset \mathbb{C}^{N}$ be a connected real-analytic submanifold. Then there exists a closed proper real-analytic subvariety $V \subset$ $M$ such that for any $p \in M \backslash V$, any real-analytic submanifold $M^{\prime} \subset \mathbb{C}^{N}$ with $\operatorname{dim}_{\mathbb{R}} M=$ $\operatorname{dim}_{\mathbb{R}} M^{\prime}$, any $p^{\prime} \in M^{\prime}$ and any positive integer $k$, if $f:\left(\mathbb{C}^{N}, p\right) \rightarrow\left(\mathbb{C}^{N}, p^{\prime}\right)$ is a formal equivalence sending $M$ into $M^{\prime}$, then there exists a convergent (biholomorphic) mapping $f_{k}:\left(\mathbb{C}^{N}, p\right) \rightarrow\left(\mathbb{C}^{N}, p^{\prime}\right)$ sending $M$ into $M^{\prime}$, whose Taylor series agrees with $f$ up to order $k$.

Theorem 1.8. (Baouendi, Mir, Rothschild [BMR02]) Let $M \subset \mathbb{C}^{N}$ be a real-analytic $C R$ submanifold. Then for any point $p \in M$ of finite type, any real-analytic CR-submanifold $M^{\prime} \subset \mathbb{C}^{N}$ with $\operatorname{dim}_{\mathbb{R}} M=\operatorname{dim}_{\mathbb{R}} M^{\prime}$, any $p^{\prime} \in M^{\prime}$ and any positive integer $k$, if $f:\left(\mathbb{C}^{N}, p\right) \rightarrow$ $\left(\mathbb{C}^{N}, p^{\prime}\right)$ is a formal equivalence sending $M$ into $M^{\prime}$, there exist a convergent (biholomorphic) mapping $f_{k}:\left(\mathbb{C}^{N}, p\right) \rightarrow\left(\mathbb{C}^{N}, p^{\prime}\right)$ sending $M$ into $M^{\prime}$, whose Taylor series agrees with $f$ up to order $k$.

We should mention that Theorem 1.7 and Theorem 1.8 hold for more general formal maps. We refer the reader to [BRZ01a] and [BMR02] for more details on this matter. In view of Theorem 1.7 and Theorem 1.8, one may formulate a more general approximation problem for formal holomorphic maps in complex spaces of (possibly) different dimensions:

Conjecture 1.9. Let $M \subset \mathbb{C}^{N}$ and $M^{\prime} \subset \mathbb{C}^{N^{\prime}}$ be real-analytic $C R$-submanifolds, $N, N^{\prime} \geq$ 2. Then for every $p \in M$, every $p^{\prime} \in M^{\prime}$, every positive integer $k$, if $f:\left(\mathbb{C}^{N}, p\right) \rightarrow\left(\mathbb{C}^{N^{\prime}}, p^{\prime}\right)$ is formal map sending $M$ into $M^{\prime}$, there exists a convergent mapping $f_{k}:\left(\mathbb{C}^{N}, p\right) \rightarrow$ $\left(\mathbb{C}^{N^{\prime}}, p^{\prime}\right)$ sending $M$ into $M^{\prime}$, whose Taylor series agrees with $f$ up to order $k$.

The authors have recently proved the following result supporting Conjecture 1.9:

Theorem 1.10 (Meylan, Mir, Zaitsev [MMZ03]). Let $M \subset \mathbb{C}^{N}$ be a real-analytic CRsubmanifold. Then for any point $p \in M$ of finite type, any real-algebraic subset $M^{\prime} \subset \mathbb{C}^{N^{\prime}}$, any $p^{\prime} \in M^{\prime}$, and any positive integer $k$, if $f:\left(\mathbb{C}^{N}, p\right) \rightarrow\left(\mathbb{C}^{N^{\prime}}, p^{\prime}\right)$ is a formal mapping sending $M$ into $M^{\prime}$, there exist a convergent mapping $f_{k}:\left(\mathbb{C}^{N}, p\right) \rightarrow\left(\mathbb{C}^{N^{\prime}}, p^{\prime}\right)$ sending $M$ into $M^{\prime}$, whose Taylor series agrees with $f$ up to order $k$.

The algebraicity of the target set $M^{\prime}$ is essential for the proof of Theorem 1.10 given in [MMZ03]. 


\section{Segre Sets technique}

We now describe a common tool needed for the proofs of Theorems 1.7, 1.8 and 1.10. It is the so-called Segre sets technique introduced by Baouendi, Ebenfelt and Rothschild [BER96, BER99a, BER03] that has been very successful for studying real-analytic and formal CR-maps between real-analytic CR-manifolds (see e.g. [BER96, BER97, Z97, BER98, BER99b, BER99a, Z99, M00, BRZ01a, BRZ01b, KZ01, La01, BMR02, M02a, M02b, MMZ03, BER03]). Roughly speaking, the Segre sets approach extends to higher codimension the Segre variety approach used by Webster [W77] for mapping problems between real hypersurfaces in complex space. We recall here some basic ideas of this approach.

Let $M \subset \mathbb{C}^{N}$ be a real-analytic generic submanifold of codimension $d$ through the origin and let $\rho(z, \bar{z}):=\left(\rho_{1}(z, \bar{z}), \ldots, \rho_{d}(z, \bar{z})\right)$ be a vector-valued real-analytic defining function of $M$ near the origin so that $\rho_{1}, \ldots, \rho_{d}$ have linearly independent complex differentials. Hence there is a connected open neighborhood $U \subset \mathbb{C}^{N}$ of the origin such that

$$
M \cap U=\{z \in U: \rho(z, \bar{z})=0\} .
$$

Complexification of the defining equation of $M$ yields the so-called complexification of $M$, which is a complex submanifold of (complex) codimension $d$ in $\mathbb{C}^{2 N}$ defined by

$$
\mathcal{M}:=\left\{(z, \zeta) \in U \times U^{*}: \rho(z, \zeta)=0\right\},
$$

where $U^{*}:=\{\bar{z}: z \in U\}$. For any $w \in U$, its Segre variety $Q_{w}$ is given by

$$
Q_{w}:=\{z \in U: \rho(z, \bar{w})=0\} .
$$

Each Segre variety $Q_{w}$ is a complex submanifold of (complex) codimension $d$ in $\mathbb{C}^{N}$. After the pioneering work of Webster [W77], Segre varieties became a basic tool for mapping problems. For real-analytic generic submanifolds of higher codimension, Baouendi, Ebenfelt and Rothschild [BER96] (see also [BER99a, BER03]) introduced the Segre sets $Q_{w}^{s}$, for $w \in M$ sufficiently close to the origin, by setting inductively $Q_{w}^{1}:=Q_{w}, Q_{w}^{s+1}:=\cup_{z \in Q_{w}^{s}} Q_{z}$ for $s \geq 1$. Another way of looking at the Segre sets was given by the third author in [Z97, Z99] through the use of the so-called iterated complexification of $M$. For every $s \geq 1$, define the iterated complexification of $M$ at 0 by setting

$$
\mathcal{M}^{s}:=\left\{\xi=\left(\xi^{0}, \ldots, \xi^{s}\right) \in \mathbb{C}^{(s+1) N}: \rho^{(j)}\left(\xi^{j-1}, \xi^{j}\right)=0 \text { for all } j=1, \ldots, s\right\},
$$

where

$$
\rho^{(j)}\left(\xi^{j-1}, \xi^{j}\right):= \begin{cases}\bar{\rho}\left(\xi^{j-1}, \xi^{j}\right) & \text { if } j \text { is even }, \\ \rho\left(\xi^{j-1}, \xi^{j}\right) & \text { if } j \text { is odd. }\end{cases}
$$

(In this definition, $\xi^{0}, \ldots, \xi^{s}$ are assumed to be sufficiently close to the origin and for a holomorphic function $h$ defined near the origin in $\mathbb{C}^{k}, \bar{h}$ denotes the holomorphic function obtained from $h$ by taking complex conjugates of the coefficients in its power series expansion.) The iterated complexification $\mathcal{M}^{s}$ is a complex submanifold of $\mathbb{C}^{(s+1) N}$ of dimension $s n+N$ where $n$ is the CR-dimension of $M$ (i.e. $N-d$ ). We can define on $\mathcal{M}^{s}$ 
two projections $\pi_{s}: \mathcal{M}^{s} \rightarrow \mathbb{C}^{N}$ and $\pi_{s}^{\prime}: \mathcal{M}^{s} \rightarrow \mathbb{C}^{N}$ on the first and last component by setting

$$
\pi_{s}\left(\xi^{0}, \ldots, \xi^{s}\right)=\xi^{0}, \text { and } \pi_{s}^{\prime}\left(\xi^{0}, \ldots, \xi^{s}\right)=\xi^{s}
$$

In this way, for $p \in M$ sufficiently close to the origin, the Segre set $Q_{p}^{s}$ coincide with the set $\pi_{s}^{\prime}\left(\pi_{s}^{-1}(\{p\})\right)$ or with its complex conjugate, depending on whether $s$ is odd or even. It is often useful to parametrize the Segre sets by the so-called iterated Segre sets mappings (see [BER99a, BER03]). Since $M$ is generic, we may assume, after possibly renumbering the coordinates, that the $d \times d$ matrix $\frac{\partial \rho}{\partial \eta}(0) \neq 0$ where $z=(\omega, \eta) \in \mathbb{C}^{n} \times \mathbb{C}^{d}$. Then, by the implicit function theorem and after shrinking $U$ if necessary, the complexification $\mathcal{M}$ of $M$ can be viewed as a graph of the form

$$
\mathcal{M}:=\left\{(z, \zeta) \in U \times U^{*}: \eta=\theta(\omega, \zeta)\right\}
$$

for some holomorphic function $\theta$. For $p \in M$ sufficiently close to the origin, define the iterated Segre mapping $v_{p}^{s}: \mathbb{C}^{s n} \rightarrow \mathbb{C}^{N}, 0 \leq s \leq 2(d+1)$, inductively as follows:

$$
v_{p}^{0}:=p, \quad v_{p}^{s+1}\left(t^{1}, \ldots, t^{s+1}\right):=\left(t^{s+1}, \theta\left(t^{s+1}, \overline{v_{p}^{s}}\left(t^{1}, \ldots, t^{s}\right)\right)\right), \quad t^{j} \in \mathbb{C}^{n} .
$$

We may assume that for each $s$ as above, the map

$$
\mathbb{C}^{N} \times \mathbb{C}^{s n} \ni\left(w, t^{1}, \ldots, t^{s}\right) \mapsto v_{w}^{s}\left(t^{1}, \ldots, t^{s}\right) \in \mathbb{C}^{N}
$$

which, with the above choice of parametrization of $\mathcal{M}$, may be identified with the map $\pi_{s}^{\prime}\left(\right.$ or $\overline{\pi_{s}^{\prime}}$ ), is holomorphic in all its variables in a sufficiently small neighborhood of the origin. Then, for $p \in M$ sufficiently small, the Segre set $Q_{p}^{s}$ can be then seen as the image of a neighborhood $\Omega_{p}^{s}$ of the point $\left(\overline{\omega_{p}}, \omega_{p}, \overline{\omega_{p}}, \omega_{p}, \ldots\right)$ in $\mathbb{C}^{s n}$ under the map $v_{p}^{s}$, where $p=\left(\omega_{p}, \eta_{p}\right)$. (Note that the Segre sets $Q_{p}^{s}$ depend on the choice of the neighborhood $\Omega_{p}^{s}$ and, unlikely Segre varieties, a choice of a smaller neighborhood yields smaller Segre sets even at the level of germs.)

From the point of view of mapping theory, regularity and uniqueness properties (such as e.g. convergence or finite jet determination of formal maps) are being "propagated" through the Segre sets. The following theorem is crucial for the proofs of Theorems 1.7, 1.8 and 1.10. It is a finite type criterion stating that Segre sets of order $2(d+1)$ attached to the origin cover an open neighborhood of 0 in $\mathbb{C}^{N}$ if only if the manifold $M$ is of finite type.

Theorem 2.1 (Baouendi, Ebenfelt, Rothschild [BER99a, BER03]). Let $M \subset \mathbb{C}^{N}$ be a real-analytic generic submanifold of codimension $d$ and CR-dimension $n$ through the origin. Then $M$ is of finite type at 0 if and only if the map $\left.\pi_{2(d+1)}^{\prime}\right|_{\pi_{2(d+1)}^{-1}(\{0\})}$ has rank $N$ at points in $\pi_{2(d+1)}^{-1}(\{0\}) \cap \pi_{2(d+1)}^{\prime-1}(\{0\})$ arbitrarily close to $0 \in \mathcal{M}^{2(d+1)}$.

\section{Algebraic approximation of analytic CR-Mappings Between REAL-ALGEBRAIC SUBMANIFOLDS}

In the category of real-algebraic submanifolds, another natural notion of equivalence, namely that of algebraic equivalence, is defined as follows. 
Definition 3.1. Given two germs $(M, p)$ and $\left(M^{\prime}, p^{\prime}\right)$ of real-algebraic submanifolds of the same dimension in $\mathbb{C}^{N}$, we say that $(M, p)$ and $\left(M^{\prime}, p^{\prime}\right)$ are algebraically equivalent if there exists a local (complex-)algebraic biholomorphism $f:\left(\mathbb{C}^{N}, p\right) \rightarrow\left(\mathbb{C}^{N}, p^{\prime}\right)$ sending $M$ into $M^{\prime}$.

Recall that a real submanifold $M \subset \mathbb{C}^{N}$ is real-algebraic if it is contained in an irreducible real-algebraic subvariety of $\mathbb{C}^{N}$ of same dimension as $M$. Also, recall that a holomorphic map is algebraic (or Nash) if each of its components satisfies a non-trivial polynomial identity with (holomorphic) polynomial coefficients. It is natural to compare the notions of biholomorphic and algebraic equivalence: Given two (germs of) real-algebraic submanifolds $(M, p)$ and $\left(M^{\prime}, p^{\prime}\right)$ of the same dimension in $\mathbb{C}^{N}$ that are biholomorphically equivalent, are they automatically algebraically equivalent?

We should mention that no example is known of a pair of biholomorphically equivalent real-algebraic submanifolds which are not algebraically equivalent. Since the techniques used in the proofs of the results mentioned in $\S 1$ have corresponding algebraic analogs (e.g. the implicit function theorem, Artin's approximation theorem [A69], etc.), Theorem 1.1 admits the following algebraic version for local holomorphic maps.

Theorem 3.2 (Baouendi, Rothschild, Zaitsev [BRZ01b]). Let $M \subset \mathbb{C}^{N}$ be a connected real-algebraic submanifold. Then there exists a closed proper real-algebraic subvariety $V \subset M$ such that for any $p \in M \backslash V$, any real-algebraic submanifold $M^{\prime} \subset \mathbb{C}^{N}$ with $\operatorname{dim}_{\mathbb{R}} M=\operatorname{dim}_{\mathbb{R}} M^{\prime}$, and any $p^{\prime} \in M^{\prime}$, the germs $(M, p)$ and $\left(M^{\prime}, p^{\prime}\right)$ are biholomorphically equivalent if and only if they are algebraically equivalent.

On the other hand, the algebraic version of Theorem 1.3 given in Theorem 3.3 below requires only the first manifold $M$ to be (connected and) of finite type somewhere, which is weaker than what is required in Theorem 1.3.

Theorem 3.3 (Baouendi, Mir, Rothschild [BMR02]). Let $M \subset \mathbb{C}^{N}$ be a connected realalgebraic $C R$-submanifold of finite type at some point. Then for any point $p \in M$, any real-algebraic CR-submanifold $M^{\prime} \subset \mathbb{C}^{N}$ with $\operatorname{dim}_{\mathbb{R}} M=\operatorname{dim}_{\mathbb{R}} M^{\prime}$, any point $p^{\prime} \in M^{\prime}$, the germs $(M, p)$ and $\left(M^{\prime}, p^{\prime}\right)$ are algebraically equivalent if and only they are biholomorphically equivalent.

Since connected real-algebraic hypersurfaces that are of finite type at some point coincide with Levi-nonflat ones, and since Levi-flat real-algebraic hypersurfaces are locally algebraically equivalent to a real hyperplane, Theorem 3.3 immediately yields the following positive answer for hypersurfaces to the question posed in the beginning of this section.

Corollary 3.4 (Baouendi, Mir, Rothschild [BMR02]). Two germs of real-algebraic hypersurfaces in $\mathbb{C}^{N}$ are biholomorphically equivalent if and only if they are algebraically equivalent. 
Note that the question whether biholomorphic equivalence of general real-algebraic submanifolds in $\mathbb{C}^{N}$ implies their algebraic equivalence remains open already for submanifolds of codimension two.

A related problem is to approximate up to any given order a local holomorphic map sending two real-algebraic submanifolds (in complex spaces of arbitrary dimension) into each other by algebraic holomorphic maps. We shall show in this paper that a suitable modification of the arguments of the proof of Theorem 1.10, in combination with some additional work, can be used to prove the following new approximation result generalizing, in particular, Theorem 3.3 (and Corollary 3.4):

Theorem 3.5. Let $M \subset \mathbb{C}^{N}$ be a connected real-algebraic CR-submanifold of finite type at some point. Then for any point $p \in M$, any real-algebraic subset $M^{\prime} \subset \mathbb{C}^{N^{\prime}}$, any point $p^{\prime} \in M^{\prime}$ and any positive integer $k$, if $f:\left(\mathbb{C}^{N}, p\right) \rightarrow\left(\mathbb{C}^{N^{\prime}}, p^{\prime}\right)$ is a local holomorphic map sending $M$ into $M^{\prime}$, there exists an algebraic holomorphic map $f_{k}:\left(\mathbb{C}^{N}, p\right) \rightarrow\left(\mathbb{C}^{N^{\prime}}, p^{\prime}\right)$ sending $M$ into $M^{\prime}$ whose Taylor series at $p$ agrees with that of $f$ up to order $k$.

In the rest of the section, we shall give the necessary modifications from the proof of [MMZ03, Theorem 1.1] leading to the proof of Theorem 3.5. Without loss of generality, we may assume that $p=p^{\prime}=0$. Moreover, it is easy to see, by using the intrinsic complexification of $M$ (see e.g. the proof of [MMZ03, Theorem 1.1]), that it is enough to show Theorem 3.5 in case $M$ is generic, which we shall assume in the following.

Similarly to [CMS99, MMZ03] (see also [Pu90a, Pu90b] in another context), to a given germ of a holomorphic map $f:\left(\mathbb{C}_{Z}^{N}, 0\right) \rightarrow \mathbb{C}_{Z^{\prime}}^{N^{\prime}}$ defined on a connected open neighborhood $U$ of the origin, associate a germ at $(0, f(0))$ of a complex-algebraic set $\mathcal{Z}_{f} \subset \mathbb{C}^{N} \times \mathbb{C}^{N^{\prime}}$ as follows. Define $\mathcal{Z}_{f}$, the (algebraic) Zariski closure of the graph of $f$, to be the smallest irreducible complex-algebraic subset containing the graph of $f$. Setting $\mu_{f}:=\operatorname{dim}_{\mathbb{C}} \mathcal{Z}_{f}$, it is clear that $\mu_{f} \geq N$ (since the graph of $f$ is contained in $\mathcal{Z}_{f}$ ). One of the interests in introducing the set $\mathcal{Z}_{f}$ lies in the fact that it contains some information about the algebraic properties of the map $f$. For instance, it is easy to see that $f$ is algebraic if and only if $\mu_{f}=N$. Another interesting fact is that one may apply directly the algebraic version of Artin's approximation theorem given in [A69] to the system of (holomorphic) polynomial equations defining $\mathcal{Z}_{f}$ to obtain, for any positive integer $k$, a local holomorphic algebraic map $f_{k}:\left(\mathbb{C}^{N}, 0\right) \rightarrow\left(\mathbb{C}^{N^{\prime}}, 0\right)$, whose graph is contained in $\mathcal{Z}_{f}$, with the same $k$-jet at the origin as that of $f$. We fix any such sequence $\left(f_{k}\right)_{k>0}$ associated to $f$ and assume that each $f_{k}$ is defined on a sufficiently small polydisc $\Delta_{k} \subset \mathbb{C}^{N}$ centered at the origin.

Our main goal now is to prove that, if $M$ satisfies the finite type condition stated in Theorem 3.5 and if $f$ sends (a neighborhood of 0 in) $M$ into $M^{\prime}$, then necessarily there exists a union of real-algebraic irreducible components of the real-algebraic subset $\mathcal{Z}_{f} \cap\left(M \times \mathbb{C}^{N^{\prime}}\right)$ that is contained in $M \times M^{\prime}$ and that contains $\Gamma_{f_{k}} \cap\left(M \times \mathbb{C}^{N^{\prime}}\right)$, for all sufficiently large $k$. Here $\Gamma_{f_{k}}$ denotes the graph of $f_{k}$ over $\Delta_{k}$. The precise statement is given by the following result: 
Proposition 3.6. Let $M, M^{\prime}, f, \mathcal{Z}_{f}$ and $\left(f_{k}\right)_{k>0}$ be as above. Then after shrinking $M$ around the origin if necessary, there exist a positive integer $k_{0}$ and an appropriate union $Z_{f}$ of real-algebraic irreducible components of the real-algebraic subset $\mathcal{Z}_{f} \cap\left(M \times \mathbb{C}^{N^{\prime}}\right)$ such that for any $k \geq k_{0}, \Gamma_{f_{k}} \cap\left(M \times \mathbb{C}^{N^{\prime}}\right) \subset Z_{f} \subset M \times M^{\prime}$.

Clearly, Theorem 3.5 follows directly from Proposition 3.6 and the fact that each $f_{k}$ is algebraic by our construction.

By inspecting now the proof of Theorem [MMZ03, Theorem 7.1 (i)] (which is somewhat analogous to the statement of Proposition 3.6 in the formal setting), it is easy to check that the proof of Proposition 3.6 can be achieved in the same way (with suitable minor modifications) using the following result (cf. [CMS99, Proposition 5.1] for a special case).

Proposition 3.7. Let $\Omega \subset \mathbb{C}^{N}$ be a connected open subset and $M \subset \Omega$ a connected realalgebraic generic submanifold that is of finite type at some point. Let $F: \Omega \rightarrow \mathbb{C}^{k}$ be a holomorphic map, $k \geq 1$, such that the components of $F$ together with their complex conjugates $\bar{F}$ satisfy on $M$ a nontrivial polynomial identity with real polynomial coefficients. Then the components of $F$ satisfy on $\Omega$ a nontrivial polynomial identity with holomorphic polynomial coefficients.

By following the arguments of [CMS99, Proposition 5.1] and [MMZ03, Proposition 4.3] (see also [Pu90a, Pu90b] where they first appear in another context), it is easy to see that Proposition 3.7 is a consequence of the following lemma:

Lemma 3.8. Let $\Omega \subset \mathbb{C}^{N}$ be a connected open subset and $M \subset \Omega$ a connected realalgebraic generic submanifold that is of finite type at some point. Let $h: \Omega \rightarrow \mathbb{C}$ and $g: \Omega \rightarrow \mathbb{C}^{m}, m \geq 1$, be holomorphic maps satisfying

$$
h(z)=\varphi(z, \overline{g(z)}), z \in M,
$$

for some holomorphic algebraic function $\varphi$ of its arguments. Then $h$ is algebraic.

Proof of Lemma 3.8. Let $d$ be the codimension of $M$ in $\mathbb{C}^{N}$ and $n$ its CR-dimension, so that $N=n+d$. It is enough to prove that $h$ is algebraic near some point $p_{0}$ of $M$ of finite type. We may choose local holomorphic algebraic coordinates $z=(\omega, \eta) \in \mathbb{C}^{n} \times \mathbb{C}^{d}$ vanishing at $p_{0}$ such that the complexification $\mathcal{M}$ of $M$ near $p_{0}$ is given by (2.4) for some holomorphic algebraic function $\theta$. Complexifying (3.1), we obtain

$$
h(z)=\varphi(z, \bar{g}(\zeta)),(z, \zeta) \in \mathcal{M} .
$$

For $0 \leq s \leq 2(d+1)$, let $v_{0}^{s}$ be the associated iterated Segre mapping of order $s$ at 0 as given by $(2.5)$. Using the fact, which follows directly from $(2.5)$, that the map $\left(v_{0}^{s+1}, \overline{v_{0}^{s}}\right)$ takes its values in $\mathcal{M}$, and using (3.2), we obtain the identity

$$
h \circ v_{0}^{s+1}=\varphi \circ\left(v_{0}^{s+1}, \bar{g} \circ \overline{v_{0}^{s}}\right),
$$

which holds for values $\left(t^{1}, \ldots, t^{s+1}\right)$ sufficiently close to the origin in $\mathbb{C}^{(s+1) n}$. We now show that the algebraicity of $h$ "propagates" along Segre sets by establishing the following claim. 
Claim. Assume that for some $1 \leq s \leq 2(d+1)$, all derivatives of $h$ pulled back via $v_{0}^{s-1}$ are algebraic. Then the same holds for all derivatives of $h$ pulled back via $v_{0}^{s+1}$.

The proof of the claim uses an argument appearing in [M98, M00, BMR02, M02a, MMZ03]. By [BMR02, Lemma 8.2], we can find a holomorphic algebraic map $\left(t^{1}, \ldots, t^{s}\right) \mapsto$ $\lambda\left(t^{1}, \ldots, t^{s}\right)$ near 0 such that

$$
\left.v_{0}^{s+1}\left(t^{1}, \ldots, t^{s}, t^{s+1}\right)\right|_{t^{s+1}=\lambda\left(t^{1}, \ldots, t^{s}\right)}=v_{0}^{s-1}\left(t^{1}, \ldots, t^{s-1}\right) .
$$

Then by differentiating infinitely many times (3.3) with respect to $t^{s+1}$, setting $t^{s+1}=$ $\lambda\left(t^{1}, \ldots, t^{s}\right)$, using (3.4), the assumption in the claim and the algebraic version of Artin's approximation theorem [A69], we obtain the existence of an algebraic map $\left(t^{1}, \ldots, t^{s}\right) \mapsto$ $G\left(t^{1}, \ldots, t^{s}\right)$ defined near the origin such $h \circ v_{0}^{s+1}=\varphi \circ\left(v_{0}^{s}, G\right)$. Since $\varphi$ is algebraic as well as the iterated Segre sets mappings, we obtain that $h \circ v_{0}^{s+1}$ is algebraic. The algebraicity of all further derivatives of $h$ pulled back via $v_{0}^{s+1}$ can be shown in the same way after differentiating first the identity (3.2) along $\mathcal{M}$. This completes the proof of the claim.

Using the fact that all derivatives of $h$ are obviously algebraic when pulled back via $v_{0}^{0}$ and using the claim, we obtain that $h \circ v_{0}^{2(d+1)}$ is algebraic. By using the finite type criterion given by Theorem 2.1 in the algebraic case (see [BER96]), we easily obtain that $h$ is algebraic. This completes the proof of Lemma 3.8 and therefore the proof of Theorem 3.5 .

\section{Convergence of formal transformations}

In $\S 1$ we have seen many situations where formal equivalence of two real-analytic submanifolds implies their biholomorphic equivalence. We shall now examine a closely related problem: Under what conditions a given formal equivalence between two submanifolds necessarily converges? Recall that it follows from the work of Chern and Moser [CM74] that any formal equivalence between two real-analytic Levi-nondegenerate hypersurfaces is convergent. For CR-submanifolds in $\mathbb{C}^{N}$, it is in general not the case as it is shown by the following examples.

Example 4.1. Let $M \subset \mathbb{C}^{N}$ be a real-analytic hypersurface through the origin and set $\widetilde{M}:=M \times \mathbb{C} \subset \mathbb{C}_{z}^{N} \times \mathbb{C}_{w}$; then for any nonconvergent formal power series $\nu(w)$ with $\nu(0)=$ 0 and $\nu^{\prime}(0) \neq 0$, the formal holomorphic map $(z, w) \mapsto(z, \nu(w))$ is a non-convergent invertible formal self map of $\widetilde{M}$.

Example 4.2. Let $M \subset \mathbb{C}_{z_{1}} \times \mathbb{C}_{z_{2}} \times \mathbb{C}_{z_{3}}$ be the real-algebraic hypersurface defined in Example 1.4. Then for any nonconvergent formal power series $\nu(z)$ with $\nu(0)=0$, the formal map $\left(z_{1}, z_{2}, z_{3}\right) \mapsto\left(z_{1} e^{\nu(z)}, z_{2} e^{-\nu(z)}, z_{3}\right)$ is a nonconvergent formal invertible self map of $M$. The difference with Example 4.1 is that there is no (germ at 0 of a) realanalytic hypersurface $\widehat{M} \subset \mathbb{C}^{2}$ such that $(\widehat{M} \times \mathbb{C}, 0)$ is biholomorphically equivalent to $(M, 0)$. However such a straightening property holds near a generic point $z_{0} \in M$.

In view of the above examples, one may therefore ask the following: Given two germs of real-analytic CR-submanifolds $(M, p)$ and $\left(M^{\prime}, p^{\prime}\right)$ in $\mathbb{C}^{N}$ of the same dimension, find 
necessary and sufficient conditions that guarantee the convergence of all their formal equivalences. An obvious necessary condition is that the source manifold is generic. In the light of Example 4.2, one should assume that the generic submanifold $M$ is not biholomorphically equivalent, near any point arbitrarily close to $p$, to a product of the form $\widehat{M} \times \mathbb{C}$ for some real-analytic CR-submanifold $\widehat{M} \subset \mathbb{C}^{N-1}$ (the submanifold $\widehat{M}$ is allowed to depend of the chosen point). This turns out to be equivalent to assume that $M$ is holomorphically nondegenerate (at $p$ ) in the sense of Stanton [S96]:

Definition 4.3. Let $M \subset \mathbb{C}^{N}$ be a real-analytic submanifold and $p \in M$. We say that $M$ is holomorphically nondegenerate at $p$ if there is no germ at $p$ of a nontrivial holomorphic vector field (with holomorphic coefficients) whose real and imaginary parts are tangent to $M$.

It is easy to see that a real-analytic hypersurface in $\mathbb{C}^{2}$ is holomorphically degenerate at some point if and only it is Levi-flat near that point (see the appendix for the definition). A less trivial fact is that a connected real-analytic CR-submanifold is holomorphically nondegenerate at a point $p \in M$ if and only if it is holomorphically nondegenerate at every point (see [BER96, BER99a]). By using flows of vector fields, Baouendi, Ebenfelt and Rothschild [BER97] observed that holomorphic nondegeneracy is necessary for the convergence of all formal equivalences sending two real-analytic generic submanifolds of the same dimension into each other. For finite type submanifolds, the sufficiency of this necessary condition was proved by the second author [M00] in the hypersurface case and by Baouendi, Rothschild and the second named author [BMR02] in higher codimension:

Theorem 4.4. (Baouendi, Mir, Rothschild [M00, BMR02]) Let $M \subset \mathbb{C}^{N}$ be a connected real-analytic generic submanifold that is holomorphically nondegenerate and of finite type (at every point). Then for any point $p \in M$, any real-analytic generic submanifold $M^{\prime} \subset$ $\mathbb{C}^{N}$ with $\operatorname{dim}_{\mathbb{R}} M=\operatorname{dim}_{\mathbb{R}} M^{\prime}$, and any point $p^{\prime} \in M^{\prime}$, any formal equivalence $f:\left(\mathbb{C}^{N}, p\right) \rightarrow$ $\left(\mathbb{C}^{N}, p^{\prime}\right)$ sending $M$ into $M^{\prime}$ is convergent.

The convergence result given by Theorem 4.4 holds also for so-called formal finite maps that are more general than formal equivalences (see [BMR02, Theorem 1.2]). Let us mention the following open problem related to Theorem 4.4:

Conjecture 4.5. Let $M \subset \mathbb{C}^{N}$ be a connected real-analytic hypersurface that is holomorphically nondegenerate. Then for any point $p \in M$, any real-analytic hypersurface $M^{\prime} \subset \mathbb{C}^{N}$, and any point $p^{\prime} \in M^{\prime}$, any formal equivalence $f:\left(\mathbb{C}^{N}, p\right) \rightarrow\left(\mathbb{C}^{N}, p^{\prime}\right)$ sending $M$ into $M^{\prime}$ is convergent.

In view of Theorem 4.4, the only remaining case to treat in order to solve Conjecture 4.5 is that of holomorphically nondegenerate real-analytic hypersurfaces that contain a complex-analytic hypersurface $S$ of $\mathbb{C}^{N}$ and the main problem in that case is to prove the convergence of the corresponding formal equivalences near any point lying on $S$.

The convergence problem for general formal mappings sending a real-analytic generic submanifold $M \subset \mathbb{C}^{N}$ into another such submanifold $M^{\prime} \subset \mathbb{C}^{N^{\prime}}$ of different dimension has 
been much less studied. One of the first results in that direction is due to Lamel [La01]. For formal maps $f:\left(\mathbb{C}^{N}, p\right) \rightarrow\left(\mathbb{C}^{N^{\prime}}, p^{\prime}\right)$ sending $M$ into $M^{\prime}$, with $M, M^{\prime}$ as above, $p \in M$, $p^{\prime} \in M^{\prime}$, an obstruction to conclude their automatic convergence is due to the possible presence of complex-analytic curves through $p^{\prime}$ contained in $M^{\prime}$; indeed, if there exists such a curve, say $(\mathbb{C}, 0) \ni t \mapsto A(t) \in\left(\mathbb{C}^{N^{\prime}}, p^{\prime}\right)$, then for any nonconvergent formal power series $\left(\mathbb{C}^{N}, p\right) \ni z \mapsto \nu(z)$ with $\nu(p)=0$, the map $z \mapsto A(\nu(z))$ is a nonconvergent formal map sending $M$ into $M^{\prime}$ (and in fact into the curve). In [MMZ03], the authors have shown that the existence of analytic curve in the target manifold is essentially the only obstruction for the convergence of formal maps in case the target manifold is real-algebraic:

Theorem 4.6 (Meylan, Mir, Zaitsev [MMZ03]). Let $M \subset \mathbb{C}^{N}$ be a real-analytic generic submanifold of finite type and $M^{\prime} \subset \mathbb{C}^{N^{\prime}}$ a real-algebraic subset with $p \in M$ and $p^{\prime} \in M^{\prime}$. Denote by $\mathcal{E}^{\prime}$ the set of all points of $M^{\prime}$ through which there exist irreducible complexanalytic subvarieties of $M^{\prime}$ of positive dimension. Then any formal (holomorphic) mapping $f:\left(\mathbb{C}^{N}, p\right) \rightarrow\left(\mathbb{C}^{N^{\prime}}, p^{\prime}\right)$ sending $M$ into $M^{\prime}$ is either convergent or sends $M$ into $\mathcal{E}^{\prime}$.

In the context of Theorem 4.6, by saying that $f$ sends $M$ into $\mathcal{E}^{\prime}$, we mean that $\varphi(f(x(t))) \equiv 0$ holds for all germs of real-analytic maps $x:\left(\mathbb{R}_{t}^{\operatorname{dim} M}, 0\right) \rightarrow(M, p)$ and $\varphi:\left(M^{\prime}, p^{\prime}\right) \rightarrow(\mathbb{R}, 0)$ such that $\varphi$ vanishes on $\mathcal{E}^{\prime}$. Such a precision is noteworthy since the subset $\mathcal{E}^{\prime}$ (that coincides with the set of infinite type points in the sense of D'Angelo) is not in general a real-analytic subset of $M^{\prime}$ as shown by the following example:

Example 4.7. Consider the tube real-algebraic hypersurface $M^{\prime} \subset \mathbb{C}^{4}$ given by

$$
\left(\operatorname{Re} z_{1}\right)^{2}-\left(\operatorname{Re} z_{2}\right)^{2}+\left(\operatorname{Re} z_{3}\right)^{2}=\left(\operatorname{Re} z_{4}\right)^{3}
$$

near the point $(1,1,0,0) \in M^{\prime}$. We claim that the subset $\mathcal{E}^{\prime} \subset M^{\prime}$ is given by $\operatorname{Re} z_{4} \geq 0$ and is therefore not analytic. (Note that $\mathcal{E}^{\prime}$ is here semi-algebraic.) Indeed, every intersection of $M^{\prime}$ with $\left\{z_{4}=\right.$ const, $\left.\operatorname{Re} z_{4} \geq 0\right\}$ contains complex lines through each point and is hence everywhere of D'Angelo infinite type. On the other hand, if $\operatorname{Re} z_{4}<0$, the coordinate $\operatorname{Re} z_{2}$ can be expressed as a strictly convex function of the other coordinates. Therefore, $M^{\prime}$ is strictly pseudoconvex at each such point and, in particular, of D'Angelo finite type.

Despite of the rather "bad" structure of the set $\mathcal{E}^{\prime}$, it is enough for the proof of Theorem 4.6 to know that it is a closed subset of $M^{\prime}$. This fact follows from the work of Lempert [Le86] and D'Angelo [D'A91]. As an immediate application of Theorem 4.6, we obtain the following characterization:

Theorem 4.8 (Meylan, Mir, Zaitsev [MMZ03]). Let $M \subset \mathbb{C}^{N}$ be a real-analytic generic submanifold of finite type and $M^{\prime} \subset \mathbb{C}^{N^{\prime}}$ a real-algebraic subset with $p \in M$ and $p^{\prime} \in M^{\prime}$. Then all formal maps $f:\left(\mathbb{C}^{N}, p\right) \rightarrow\left(\mathbb{C}^{N^{\prime}}, p^{\prime}\right)$ sending $M$ into $M^{\prime}$ are convergent if and only if $M^{\prime}$ does not contain any irreducible complex-analytic subvariety of positive dimension through $p^{\prime}$.

It is not known whether Theorem 4.8 still holds with $M^{\prime}$ being merely a real-analytic subset of $\mathbb{C}^{N^{\prime}}$. For the case of real hypersurfaces in the equidimensional case $N=N^{\prime}$, 
it has been previously known that the algebraicity condition on the target manifold in Theorem 4.8 can be dropped provided the source is also of D'Angelo finite type:

Theorem 4.9 (Baouendi, Ebenfelt, Rothschild [BER00a]). Let $M, M^{\prime} \subset \mathbb{C}^{N}$ be realanalytic hypersurfaces which do not contain nontrivial complex-analytic subvarieties. Then any formal mapping sending $M$ into $M^{\prime}$ is convergent.

Another intermediate interesting setting to study is that of formal maps (or embeddings) sending real-analytic strongly pseudoconvex hypersurfaces (in different dimensions) into each other. In case $N^{\prime}=N+1$ we have the following:

Theorem 4.10 (Mir [M02b]). Any formal embedding sending a real-analytic strongly pseudoconvex hypersurface $M \subset \mathbb{C}^{N}$ into another such hypersurface $M^{\prime} \subset \mathbb{C}^{N+1}$ is convergent.

Recall that, by a formal embedding sending $M$ into $M^{\prime}$, we mean a formal map for which the induced differential on the tangent spaces of the reference points is injective. It turns out that Theorem 4.10 is a special case of a more general result for so-called CR-transversal formal maps between Levi-nondegenerate real-analytic hypersurfaces. A formal map $F:\left(\mathbb{C}^{N}, p\right) \rightarrow\left(\mathbb{C}^{N^{\prime}}, p^{\prime}\right)$ sending two (germs of) real-analytic hypersurfaces $M$ and $M^{\prime}$ into each other is called CR-transversal if $d F(p)\left(\mathbb{C} T_{p} M\right) \not \subset T_{p^{\prime}}^{1,0} M^{\prime} \oplus T_{p^{\prime}}^{0,1} M^{\prime}$. It is easy to see that CR-transversality automatically holds if both $M$ and $M^{\prime}$ are strongly pseudoconvex and $F$ is an embedding. Hence Theorem 4.10 follows immediately from the following.

Theorem 4.11 (Mir [M02b]). Any formal CR-transversal map sending a real-analytic Levi-nondegenerate hypersurface $M \subset \mathbb{C}^{N}$ into another such hypersurface $M^{\prime} \subset \mathbb{C}^{N+1}$ is convergent.

It is noteworthy to mention that Theorem 4.11 is sharp in the sense that it can not be extended to formal CR-transversal maps with $N^{\prime}-N>1$, as shown by the following simple example (cf. [La01]):

Example 4.12. For any $N \geq 2$, consider the Levi-nondegenerate real-algebraic hypersurfaces through the origin given by

$$
\begin{aligned}
& M_{0}:=\left\{\left(z_{1}, \ldots, z_{N}\right) \in \mathbb{C}^{N}: \operatorname{Re} z_{1}=\left|z_{2}\right|^{2}+\ldots\left|z_{N}\right|^{2}\right\} \subset \mathbb{C}^{N}, \\
& M_{0}^{\prime}:=\left\{\left(z_{1}^{\prime}, \ldots, z_{N+1}^{\prime}, z_{N+2}^{\prime}\right) \in \mathbb{C}^{N+2}: \operatorname{Re} z_{1}^{\prime}=\left|z_{2}^{\prime}\right|^{2}+\ldots+\left|z_{N+1}^{\prime}\right|^{2}-\left|z_{N+2}^{\prime}\right|^{2}\right\} \subset \mathbb{C}^{N+2} .
\end{aligned}
$$

Then any nonconvergent formal power series $\nu(z)$, with $\nu(0)=0$, yields a nonconvergent formal embedding $F_{\nu}$ sending $M_{0}$ into $M_{0}^{\prime}$ defined by setting $F_{\nu}(z):=\left(z_{1}, \ldots, z_{N}, \nu(z), \nu(z)\right)$.

Example 4.12 also shows that the result of Chern-Moser mentioned in the beginning of this paragraph cannot be extended to formal embeddings of Levi-nondegenerate hypersurfaces if $N^{\prime}-N>1$. 
In addition to the result in the strongly pseudoconvex case mentioned in Theorem 4.10, Theorem 4.11 also provides a convergence result for formal embeddings in other new situations, such as e.g. when the target hypersurface is foliated by complex curves. The proof of Theorems 4.11 and 4.10 is based on Huang's work on algebraicity of local holomorphisms of real-algebraic hypersurfaces [Hu94] as well on some convergence arguments from [M00, M02a] involving the Segre sets technique mentioned in $\S 1$.

\section{APPENDIX}

A $\left(\mathcal{C}^{\infty}\right)$ smooth submanifold $M \subset \mathbb{C}^{N}$ is called a CR-submanifold if for any $p \in M$ the complex tangent space $T_{p}^{c} M:=T_{p} M \cap J\left(T_{p} M\right) \subset T_{p} M$ has constant dimension. (Here, $J: \mathbb{C}^{N} \rightarrow \mathbb{C}^{N}$ denotes the standard complex structure given by multiplication by $i$.) It is called generic if $T_{p} M+J\left(T_{p} M\right)=T_{p} \mathbb{C}^{N}$, for all $p \in M$. If $M$ is a CR-submanifold of $\mathbb{C}^{N}$, the subbundle $T^{c} M \subset T M$ induces a subbundle $T^{0,1} M$ of the complexified tangent bundle $\mathbb{C} T M:=T M \otimes \mathbb{C}$ defined on each fiber by setting $T_{p}^{0,1} M:=\left\{X+i J X: X \in T_{p}^{c} M\right\}$ for $p \in M$. Any section of $T^{0,1} M$ is usually referred to as a $(0,1)$ vector field (tangent to $M)$. The bundle $T^{0,1} M$ satisfies the conditions (4.1) and (4.2) below, that may be used to define an abstract CR-structure on any given manifold.

We say that a smooth real manifold $M$ is a CR-manifold if there exists a subbundle $\mathbb{L}$ of $\mathbb{C} T M$ (often called the CR-bundle) satisfying the following conditions

$$
\mathbb{L} \cap \overline{\mathbb{L}}=\{0\},
$$

which is the abbreviation of the following property: if $V \in \Gamma(\mathbb{L}) \cap \Gamma(\overline{\mathbb{L}})$ then $V=0$, and the following integrability condition

$$
[\mathbb{L}, \mathbb{L}] \subset \mathbb{L},
$$

which is the abbreviation of the following property: if $V, W \in \Gamma(\mathbb{L})$, then $[V, W] \in \Gamma(\mathbb{L})$. (Here if $E$ is a vector bundle over some manifold $X, \Gamma(E)$ denotes the space of smooth sections from $X$ to $E$.) The complex dimension of the bundle $\mathbb{L}$ is called the CR-dimension of $M$. Given two CR-manifolds $M, M^{\prime}$ and a map $f: M \rightarrow M^{\prime}$ of class $\mathcal{C}^{1}$, we say that $f$ is $\mathrm{CR}$ if the differential of $f$ maps $T^{c} M$ into $T^{c} M^{\prime}$ in such a way that it commutes with $J$ on $T^{c} M$. The map $f$ is called a CR-diffeomorphism if it is a diffeomorphism and $f^{-1}$ is $\mathrm{CR}$.

Let $M$ be a CR-manifold of dimension $2 n+d$, where $n=\operatorname{dim}_{C R} M$; let $\mathbb{L}$ be its CRbundle. We recall that the Levi-form of $M$ is given at a point $p \in M$ by the vector-valued hermitian form $\mathcal{L}_{p}: \mathbb{L}_{p} \times \mathbb{L}_{p} \rightarrow \mathbb{C} T_{p} M /\left(\mathbb{L}_{p} \oplus \overline{\mathbb{L}}_{p}\right)$ defined by setting

$$
\mathcal{L}_{p}\left(V_{1}(p), V_{2}(p)\right):=\frac{1}{2 i} \pi_{p}\left(\left[V_{1}, \overline{V_{2}}\right](p)\right),
$$

where $V_{1}, V_{2} \in \Gamma(M, \mathbb{L}), \mathbb{L}_{p}$ denotes the fiber of $\mathbb{L}$ at $p$ and $\pi_{p}: \mathbb{C} T_{p} M \rightarrow \mathbb{C} T_{p} M /\left(\mathbb{L}_{p} \oplus\right.$ $\left.\overline{\mathbb{L}}_{p}\right)$ is the natural projection. The CR-manifold $M$ is called Levi-nondegenerate at $p$ if $\mathcal{L}_{p}\left(v_{1}, v_{2}\right)=0$ for all $v_{1} \in \mathbb{L}_{p}$ implies $v_{2}=0$. For instance, a model case of Levinondegenerate real hypersurfaces in complex space is given by the hyperquadric $\mathbb{H}^{l+k} \subset$ 
$\mathbb{C}^{l+k+1}$ defined by

$\mathbb{H}^{l+k}:=\left\{\left(z_{1}, \ldots, z_{l+k+1}\right) \in \mathbb{C}^{l+k+1}: \operatorname{Im} z_{l+k+1}=\left|z_{1}\right|^{2}+\ldots+\left|z_{l}\right|^{2}-\left|z_{l+1}\right|^{2}-\ldots-\left|z_{l+k}\right|^{2}\right\}$.

When $k=0, \mathbb{H}^{l}$ is the standard Heisenberg hypersurface, which is a model case of real hypersurfaces that are strongly pseudoconvex i.e. for which the Levi form (which is then a hermitian form) is definite positive (or negative). On the opposite side of strongly pseudoconvex real hypersurfaces, the real hypersurfaces for which the Levi form vanishes at every point are called Levi-flat. Such real hypersurfaces, that are moreover real-analytic, are locally biholomorphically equivalent to a real hyperplane (see e.g. [BER99a]).

\section{REFERENCES}

[A68] Artin, M. - On the solutions of analytic equations. Invent. math. 5 (1968), 277-291.

[A69] Artin, M. - Algebraic approximation of structures over complete local rings. Inst. Hautes Etudes Sci. Publ. Math. 36, (1969), 23-58.

[BER96] Baouendi, M.S.; Ebenfelt, P.; Rothschild, L.P. - Algebraicity of holomorphic mappings between real algebraic sets in $\mathbb{C}^{n}$. Acta Math. 177 (1996), 225-273.

[Ber97] Baouendi, M.S.; Ebenfelt, P.; Rothschild, L.P. — Parametrization of local biholomorphisms of real analytic hypersurfaces. Asian J. Math. 1 (1997), 1-16.

[Ber98] Baouendi, M.S.; Ebenfelt, P.; Rothschild, L.P. - CR automorphisms of real analytic manifolds in complex space. Comm. Anal. Geom., 6, (1998), 291-315.

[BER99a] Baouendi, M.S.; Ebenfelt, P.; Rothschild, L.P. - Real Submanifolds in Complex Space and Their Mappings. Princeton Math. Series 47, Princeton Univ. Press, 1999.

[BER99b] Baouendi, M.S.; EBenfelt, P.; Rothschild, L.P. — Rational dependence of smooth and analytic CR mappings on their jets. Math. Ann. 315 (1999), 205-249.

[BER00a] Baouendi, M.S.; EBenfelt, P.; Rothschild, L.P. - Convergence and finite determination of formal CR mappings. J. Amer. Math. Soc. 13 (2000), 697-723.

[BER00b] Baouendi, M.S.; EBenfelt, P.; Rothschild, L.P. — Local geometric properties of real submanifolds in complex space, Bull. Amer. Math. Soc., 37, (2000) 309-336.

[BER03] Baouendi, M.S.; Ebenfelt, P.; Rothschild, L.P. - Dynamics of the Segre varieties of a real submanifold in complex space, J. Algebraic Geom., 12 (2003), 81-106.

[BMr02] Baouendi, M.S.; Mir, N; Rothschild, L.P. - Reflection ideals and mappings between generic submanifolds in complex space, J. Geom. Anal., 12, no. 4, (2002), 543-580.

[BRZ01a] Baouendi, M.S.; Rothschild, L.P.; Zaitsev, D. - Equivalences of real submanifolds in complex space. J. Differential Geom. 59 (2001), 301-351.

[BRZ01b] Baouendi, M.S.; Rothschild, L.P.; Zaitsev, D. - Points in general position in realanalytic submanifolds in $\mathbb{C}^{N}$ and applications. Ohio State Univ. Math. Res. Inst. Publ., 9, (2001), 1-20.

[BN90] Bell, S.R.; Narasimhan, R. - Proper holomorphic mappings of complex spaces, Several Complex Variables (Heidelberg) (Barth, W., Narasimhan, R., Eds.), EMS, 69, SpringerVerlag, 1990.

[BG77] Bloom, T.; Graham, I. - On type conditions for generic real submanifolds of $\mathbb{C}^{n}$. Invent. math. 40 (1977), 217-243.

[C32a] CARTAn, E. - Sur la géométrie pseudo-conforme des hypersurfaces de deux variables complexes, Part I, Ann. Math. Pura Appl. 11, (1932), 17-90.

[C32b] CARTAn, E. - Sur la géométrie pseudo-conforme des hypersurfaces de deux variables complexes, Part II, Ann. Sc. Norm. Sup. Pisa 1, (1932), 333-354. 
[CM74] Chern, S.S; Moser, J.K. - Real hypersurfaces in complex manifolds. Acta Math. 133 (1974), 219-271.

[CMS99] Coupet, B.; Meylan, F.; Sukhov, A. - Holomorphic maps of algebraic CR manifolds. Internat. Math. Res. Not. 1 (1999), 1-29.

[D'A91] D'Angelo, J.P. - Finite type and the intersection of real and complex subvarieties. Several complex variables and complex geometry, Part 3 (Santa Cruz, CA, 1989), 103-117, Proc. Sympos. Pure Math. 52, Part 3, Amer. Math. Soc., Providence, RI, 1991.

[Fo93] Forstnerič, F. - Proper holomorphic mappings: a survey. Several complex variables (Stockholm, 1987/1988), 297-363, Math. Notes 38, Princeton Univ. Press, Princeton, NJ, 1993.

[G02] GonG, X. - Existence of real-analytic surfaces with hyperbolic complex tangent that are formally but not holomorphically equivalent to quadrics, Indiana Univ. Math. J., (to appear).

[Hu94] HuANG, X. - On the mapping problem for algebraic real hypersurfaces in the complex spaces of different dimensions. Ann. Inst. Fourier, (Grenoble) 44, no. 2, 433-463 (1994).

[Hu01] HuAng, X. - On some problems in several complex variables and CR geometry. First International Congress of Chinese Mathematicians (Beijing, 1998), 383-396, AMS/IP Stud. Adv. Math. 20, Amer. Math. Soc., Providence, RI, 2001.

[KZ01] KIM, S.-Y.; ZAitsev, D. - The equivalence and embeddings problems for CR structures of any codimension (preprint 2001).

[K72] KoHN, J.J. - Boundary behavior of $\bar{\partial}$ on weakly pseudo-convex manifolds of dimension two. J. Differential Geom. 6, (1972), 523-542.

[La01] Lamel, B. - Holomorphic maps of real submanifolds in complex spaces of different dimensions. Pacific J. Math. 201 (2001), no. 2, 357-387.

[Le86] Lempert, L. - On the boundary behavior of holomorphic mappings. Contributions to several complex variables, 193-215, Aspects Math., E9, Viehweg, Braunschweig, 1986.

[M98] Mir, N. - Germs of holomorphic mappings between real-algebraic hypersurfaces, Ann. Inst. Fourier, (Grenoble) 48 no. 4, (1998), 1025-1043.

[M00] Mir, N. - Formal biholomorphic maps of real analytic hypersurfaces. Math. Res. Lett. 7 (2000), 343-359.

[M02a] Mir, N. - On the convergence of formal mappings. Comm. Anal. Geom. 10, no. 1, (2002), $23-59$.

[M02b] MiR, N. - Convergence of formal embeddings between real-analytic hypersurfaces in codimension one, J. Differential Geom. 62 (2002), 163-173.

[MMZ03] Meylan, F.; Mir, N.; Zaitsev, D. - Approximation and convergence of formal CRmappings. Internat. Math. Res. Notices 4, (2003), 211-242.

[MW83] Moser, J.K.; Webster, S.M. - Normal forms for real surfaces in $\mathbb{C}^{2}$ near complex tangents and hyperbolic surface transformations. Acta Math. 150 (1983), no. 3-4, 255-296.

[N62] NAgAno, T. - Linear differential systems with singularities and an application to transitive Lie algebras. J. Math. Soc. Japan 14, (1962), 397-429.

[Po07] Poincaré, H. - Les fonctions analytiques de deux variables et la représentation conforme, Rend. Circ. Mat. Palermo 23, (1907), 185-220.

[Pu90a] Pushnikov, A.Yu. - Holomorphicity of CR-mappings into a space of large dimension. Mat. Zametki 48 (1990), no. 3, 147-149.

[Pu90b] Pushnikov, A.Yu. - On the holomorphy of CR-mappings of real analytic hypersurfaces. Complex analysis and differential equations, 76-84, Bashkir. Gos. Univ., Ufa, 1990.

[R03] Rothschild, L.P. - Mappings of real submanifolds in complex space, Contemp. Math., Amer. Math. Soc. (to appear).

[S96] Stanton, N. - Infinitesimal CR automorphisms of real hypersurfaces, Amer. J. Math. 118, (1996), 209-233. 
[Ta62] TANAKA, N. - On the pseudo-conformal geometry of hypersurfaces of the space of $n$ complex variables J. Math. Soc. Japan, 14, 397-429, (1962).

[Tr00] Treves, F. - A treasure trove of geometry and analysis: the hyperquadric, Notices Amer. Math. Soc. 47, no. 10, (2000), $1246-1256$.

[V85] Vitushkin, A.G. - Holomorphic mappings and the geometry of hypersurfaces, Encyclopedia of Mathematical Sciences, Vol. 7, Several Complex Variables I, Springer-Verlag, Berlin, 1985, pp. 159-214.

[W77] WeBster, S. - On the mapping problem for algebraic real hypersurfaces. Invent. math., 43, (1977), 53-68.

[Z97] ZAITSEV, D. - Germs of local automorphisms of real analytic CR structures and analytic dependence on $k$-jets. Math. Res. Lett. 4 (1997), 823-842.

[Z99] ZAitsev, D. - Algebraicity of local holomorphisms between real-algebraic submanifolds in complex space, Acta Math., 183, (1999), 273-305.

[Z02] Zaitsev, D. - Unique jet determination of local CR-maps by their jets: a survey, Atti Accad. Naz. Lincei Cl. Sci. Fis. Mat. Natur. Rend. Lincei (9) Mat. Appl., 13, (2002), 295-305.

F. Meylan: Institut de Mathématiques, Université de Fribourg, 1700 Perolles, FriBOURG, SWITZERLAND

E-mail address: francine.meylan@unifr.ch

N. Mir: Université de Rouen, Laboratoire de Mathématiques Raphä̈l Salem, UMR 6085 CNRS, 76821 Mont-Saint-Aignan Cedex, France

E-mail address: Nordine.Mir@univ-rouen.fr

D. Zaitsev: School of Mathematics, Trinity College, Dublin 2, Ireland

E-mail address: zaitsev@maths.tcd.ie 